\title{
Senza voce e senza nome. Il peace journalism come prospettiva per valutare la copertura informativa italiana dei migranti
}

In un saggio del 2007 il giornalista David Loyn - per decenni corrispondente della BBC - definiva il peace journalism come "perfettamente inutile nel migliore dei casi, nel peggiore come un insieme di prescrizioni fuorvianti per il giornalismo" (Loyn, 2007: 2). Riconosciamo dunque, da estimatori di Loyn, che partire proprio dal peace journalism per compiere un'analisi sulla copertura informativa del giornalismo professionale italiano sui fenomeni migratori è almeno scivoloso. $\mathrm{Ci}$ ripariamo dietro la consapevolezza che quell'articolo, pur provenendo da una fonte autorevole, non ha ricevuto un'accoglienza straordinaria né dalla comunità scientifica né dal mondo giornalistico.

Nella contemporanea ecologia dei media, contraddistinta dalla molteplicità di attori, dall'abbondanza di flussi comunicativi (Van Aelst et al., 2017) e dalla loro ibridazione (Chadwick, 2013), i giornalisti svolgono ancora un importante ruolo di selezionatori delle notizie, delle occorrenze e dei fatti da valorizzare nel racconto delle stesse, permettendo a certi aspetti della realtà di emergere e impedendo la diffusione di altri (Godler e Reich, $2017)^{1}$. Insomma, nonostante la complessità della ecologia dei media contemporanea, il giornalismo rimane un discorso performativo finalizzato a imporre e legittimare una valida rappresentazione del mondo e a creare distinzioni (Pogliano, 2011), ma quel potere e quell'autorevolezza eventualmente accordati per raccontare accadimenti, storie rilevanti «sono negoziati, contingenti, mutevoli e prendono sembianze diverse nel tempo» (Splendore, 2017: 12-13). Proprio per questo motivo, nella crescente importanza che riveste il tema dell'immigrazione nel contemporaneo sistema dei media e nella produzione giornalistica (si veda fra gli altri Bruno, 2014; Binotto et al, 2016; Pogliano e Ponzo, 2019), ci appare opportuno proporre come chiave di lettura di questa copertura il peace journalism, che sta incontrando un rinnovato interesse nel più ampio contesto dei journalism studies (cfr. Kalfeli e Frangonikolopoulos, 2019). L'opportunità di utilizzare il peace journalism come prospettiva per valutare la produzione giornalistica italiana sull'immigrazione appoggia su una considerazione e una evidenza.

\footnotetext{
${ }^{1}$ Per una discussione su questo tema relativa allo specifico contesto del peace journalism si veda Lynch (2017).
} 
La considerazione è che molta letteratura scientifica, e non ${ }^{2}$, stia focalizzando la propria attenzione proprio sulle modalità di copertura dell'immigrazione, anche per i suoi risvolti in chiave politica ed elettorale (si pensi, in Italia, al ruolo degli avvenimenti di Macerata durante la campagna elettorale del 2018, cfr. Bentivegna e Natale, 2019), ma manca una prospettiva adeguata per valutarla. L'altro aspetto, ancor più determinante, è il fatto che l'Associazione Carta di Roma abbia redatto un protocollo deontologico ${ }^{3}$ che invita i giornalisti italiani ad "osservare la massima attenzione nel trattamento delle informazioni concernenti i richiedenti asilo, i rifugiati, le vittime della tratta ed i migranti nel territorio della Repubblica Italiana ed altrove", una serie di prescrizioni rispetto al trattamento di coloro che in letteratura sono definiti con l'acronimo di RASIM (rifugiati, richiedenti asilo - asylum seekers -, immigrati e migranti). La Carta di Roma, per quanto non contenga un riferimento esplicito al peace journalism, ne richiama molti dei punti fondamentali.

Nel prossimo paragrafo questo articolo introduce e discute gli aspetti fondamentali legati al peace journalism. Il paragrafo successivo presenta invece una rassegna della letteratura delle più recenti ricerche cha hanno indagato il modo in cui il tema dell'immigrazione è stato trattato dai media. Considerata la grande mole di lavori in questo campo, si sono selezionati quelli che più di altri hanno influenzato il lavoro empirico qui presentato, sia nella sua impostazione sia nella lettura dei dati. A seguito di questa trattazione, l'articolo presenterà brevemente il metodo di ricerca adottato per analizzare la copertura data al tema dell'immigrazione durante eventi accaduti dal 2015 al 2018 da due dei principali giornali italiani (Corriere della Sera e La Stampa). Dopo la presentazione dei dati, una loro discussione sarà appunto effettuata rispetto all'ideologia giornalistica del peace journalism.

\section{Da dove nasce il peace journalism}

Thomas Hanitzsch (2007a) nella sua proposta non solo di definizione di cultura giornalistica, ma anche di precisa operativizzazione del concetto,

\footnotetext{
${ }^{2}$ Si consideri, tra le altre cose, l'introduzione al IV Rapporto Associazione Carta di Roma in cui Ilvo Diamanti sostiene che «negli ultimi anni gli immigrati e l'immigrazione, assai più del paese, hanno 'invaso' i media, al punto che sono diventati un luogo comune dell'informazione quotidiana (...) Insomma, sono divenuti un tema dominante e ricorrente, di cronaca e di dibattito pubblico» (Diamanti, 2016: 9).

${ }^{3}$ https://www.fnsi.it/upload/9b/9bf31c7ff062936a96d3c8bd1f8f2ff3/98794363d44ef430f 8e940ba93b27ee7.pdf
} 
distingue tre diverse componenti: il ruolo istituzionale che $\mathrm{i}$ giornalisti ricoprono, la concezione della realtà che i giornalisti mantengono proprio da un punto di vista epistemologico e infine gli orientamenti etici. La combinazione di orientamenti assunta in tutti o in alcuni di questi diversi aspetti della professione dà origine a quella che l'autore definisce come ideologia giornalistica.

Per Hanitzsch (2007b: 370) le ideologie formano modi internamente coerenti di valori, orientamenti e predisposizioni. Una condivisa ideologia rappresenta il cemento culturale $^{4}$ che tiene assieme il giornalismo come professione e che permette così di definirlo e identificarlo.

Le ideologie professionali nel giornalismo possono perciò essere comprese come le cristallizzazioni di distinte varietà di valori, orientamenti e predisposizioni relative al giornalismo che si articolano come una cultura professionale dominante (per esempio il cosiddetto giornalismo obiettivo) o come un set di valori contro-egemonico (Hanitzsch 2007a: 371).

Quando parla di valori contro-egemonici l'autore fa esplicitamente riferimento a una prospettiva critica che contrasta aspetti dominanti e egemonici della professione. Il giornalismo obiettivo è da considerarsi quello seguito, professato e praticato dalla maggior parte dei giornalisti proprio nelle culture giornalistiche non solo più capaci di imporre una definizione condivisa della professione, ma anche di trasmetterla altrove con quel set di valori e predisposizioni (vedi Waisbord, 2013). Quello che sostiene Hanitzsch (2007a) è che esistono anche orientamenti diversi e non di rado oppositivi. Tra questi include proprio il peace journalism come ideologia professionale contro-egemonica, ovvero un modo differente di produrre e fare giornalismo. Lo identifica come un giornalismo che predilige le prese di posizione a discapito di un atteggiamento neutrale, distaccato e obiettivo, che ignora le esigenze del mercato (detto diversamente, non produce quello che i lettori hanno voglia di leggere per favorire tra loro una maggior circolazione di notizie, bensì quello che i lettori dovrebbero leggere) e che da un punto di vista epistemologico assume l'impossibilità di tradurre il reale in resoconti giornalistici mimetici (ossia in resoconti esattamente congruenti e non distinguibili dalla complessità del reale) e proprio per questo motivo un giornalismo dedito alla raccolta del maggior numero possibile di punti di vista, prospettive, evidenze che possano imprimere forza alle ricostruzioni fornite.

\footnotetext{
${ }^{4}$ Hanitzsch prende questa espressione dal lavoro di Mark Deuze (2005) intitolato Cos'è il giornalismo?
} 
Pre-Print version dell'articolo - Garusi, D., Kalfeli, N., \& Splendore, S. (2020). Senza

nome, senza voce. Il peace journalism come prospettiva per valutare la copertura

informativa italiana dei migranti. MONDI MIGRANTI. https://doi.org/10.3280/MM2020-

$\underline{002005}$

Nel complessivo quadro offerto da Hanitzsch (2007a), le ideologie professionali, come il peace journalism, occupano posizioni specifiche nello spazio di una data cultura giornalistica e si articolano e interfacciano con le altre ideologie giornalistiche rilevanti. La cultura giornalistica di un dato contesto (facilmente identificabile con i confini nazionali, soprattutto per i paesi come l'Italia in cui le minoranze linguistiche occupano spazi limitati e circoscritti) è dunque molto di più che l'insieme delle ideologie professionali presenti, è bensì l'arena in cui diverse ideologie confliggono per una interpretazione dominante della funzione sociale e dell'identità del giornalismo.

L'ideologia professionale del peace journalism nasce all'interno di una precisa categoria di giornalisti, gli inviati di guerra (Hanitzsch, 2007b), non emerge perciò in contesti relativi alla copertura dei movimenti migratori. È sollecitata proprio da quei professionisti che cominciarono a domandarsi con quali criteri avrebbero dovuto rendere conto delle atrocità dei conflitti, ma soprattutto come avrebbero potuto contribuire per una loro soluzione. Contemporaneamente, alcuni studiosi - estranei alla realtà dei journalism studies - promuovevano una visione delle pratiche giornalistiche finalizzate alla copertura informativa dalle zone di guerra capace di oltrepassare il giornalismo mainstream e i suoi valori di oggettività, neutralità e obiettività. E proprio a cavallo di queste due spinte, una più interna alla professione, l'altra che emerge in ambito accademico, che si pongono le basi per quello che (ri)conosciamo come peace journalism (Hanitzsch, 2007b).

Considerata la portata e l'ambizione di una tale proposta editoriale, studiosi e professionisti, come dimostrato anche dalle parole di Loyn (2007), non sono ancora riusciti a trovare una definizione unanimemente condivisa di peace journalism (Hanitzsch, 2007b). Uno dei motivi principali della frammentarietà delle posizioni si fa risalire proprio al ruolo accordato non solo al giornalismo, ma agli stessi media: qual è il ruolo dei media nella società? Quali sono i precipui obiettivi del giornalismo? E ancora, è possibile assegnare al giornalismo una tale responsabilità? Alcuni critici dell'introduzione di questa ulteriore ideologia giornalistica la incorniciano all'interno di orientamenti già consolidati, quali quello dell'advocacy journalism (Bratic et al., 2008), vivace anche nella cultura giornalistica italiana. Insomma, secondo questi osservatori, le disposizioni del peace journalism non sono nulla di nuovo, bensì rappresentano un'ideologia già ben consolidata in diverse culture giornalistiche del mondo. 
Il termine peace journalism è stato introdotto alla fine degli anni Sessanta da John Galtung ${ }^{5}$ per descrivere un giornalismo focalizzato sulle cause e sulle possibili soluzioni dei conflitti, un approccio giornalistico intrinsecamente normativo, che non si limitasse a raccontare le evoluzioni dei conflitti. In un articolo di molto successivo Galtung (1998) individua due modi attraverso cui i giornalisti raccontano i conflitti: la "low road" e la "high road". Attraverso la "low road", predominante nei media, il giornalismo racconta il conflitto come una battaglia o come un'arena sportiva, in cui le parti combattono per raggiungere i propri obiettivi. Il modo di narrare i fatti è quello tipicamente militare: c'è chi avanza e chi capitola, mancando l'obiettivo; le perdite vengono contate in termini di numero di morti o feriti e materiale danneggiato (Galtung, 1998). In questo tipo di giornalismo la violenza sembra essere un'opzione ragionevole e spesso appare come l'unica soluzione (McGoldrick, 2006).

Con la definizione di high-road, Galtung (1998) cesella i confini dentro cui si muove il peace journalism. Questo tipo di rappresentazione valorizza le molteplici parti in gioco, la complessità delle cause del conflitto e le situazioni in cui tutte le parti coinvolte possono uscirne vincitrici (McGoldrick, 2006). Il focus è sul complessivo contesto culturale e strutturale in cui i conflitti hanno luogo (Bratic et al., 2008) prestando particolare attenzione alla loro trasformazione (Galtung, 1998). Peleg (2006) suggella questo approccio sostenendo che incentrare gli articoli sulle conseguenze negative e immediatamente osservabili del conflitto può portare a una recrudescenza del conflitto. Consiglia, invece, di adottare uno sguardo più ampio ed ecumenico,

Nell'insieme di questo vivace dibattito, è lo stesso Hanitzsch a fornire una delle definizioni più ricorrenti (e condivise) di peace journalism descrivendolo come un giornalismo socialmente responsabile che contribuisce al processo di creazione e mantenimento della pace attraverso la proposta di una risoluzione non violenta dei conflitti (Hanitzsch, 2004) e che sollecita un costante esame critico dello stato attuale delle rappresentazioni mediali degli stessi (Hanitzsch, 2007b). Questo duplice aspetto proposto da Hanitzsch è decisivo. L'ideologia professionale del peace journalism si concentra sull'importanza di una riflessione sulle modalità di rappresentazione (non solo della redazione per la quale si lavora, ma anche del restante panorama dei media).

\footnotetext{
${ }^{5}$ Galtung assieme alla collega norvegese Mari Ruge (1965) sono ricordati all'interno dei journalism studies soprattutto per la loro intuizione rispetto ai cosiddetti news values, i valori notizia, cioè le caratteristiche che rendono notiziabile un fatto.
} 
Pre-Print version dell'articolo - Garusi, D., Kalfeli, N., \& Splendore, S. (2020). Senza nome, senza voce. Il peace journalism come prospettiva per valutare la copertura informativa italiana dei migranti. MONDI MIGRANTI. https://doi.org/10.3280/MM2020$\underline{002005}$

La proposta di una definizione più precisa e meglio adattabile in un contesto di ricerca (o di valutazione del contenuto) è dovuta a Kalfeli (2018, 2019). Nella scia della differenziazione dovuta a Galtung (1998) di low-road e high-road e con uno sforzo esplicitato di adattare la cornice del peace journalism anche alla valutazione della produzione di articoli relativi all'immigrazione, Kalfeli $(2018,2019)$ propone quello che definisce come peace frame, un insieme di precipui requisiti che caratterizzano il peace journalism e lo distinguono da cornici più conflittuali.

Tab.1 - Modello Conflict Frame-Peace Frame

\begin{tabular}{|c|c|}
\hline Conflict frame & Peace frame \\
\hline Assenza della voce dei migranti & Presenza della voci dei migranti \\
\hline Discorso costruito su due parti (noi e loro) & Un approccio pluralista \\
\hline Gli immigrati sono il problema & Il problema è la violenza \\
\hline Focus sulla disputa politica & Focus sulla cooperazione \\
\hline Uso di stereotipi & Decostruzione degli stereotipi \\
\hline Attenzione agli eventi negativi & Attenzione sulle storie degli esseri umani \\
\hline $\begin{array}{l}\text { Linguaggio emotivo (demonizzazione, } \\
\text { vittimizzazione, divisione) }\end{array}$ & Linguaggio neutrale \\
\hline Presenza di fotografie stereotipiche & Mancanza di fotografie stereotipiche \\
\hline
\end{tabular}

L'atteggiamento precauzionale e proattivo del peace journalism nel trattare i contesti di conflitto e la copertura informativa sui movimenti migratori si fondano sulla certezza che il modo in cui essi sono rappresentati dai media sia un fattore determinante per i successivi esiti (Kalfeli e Frangonikolopoulos, 2019). Come sostiene Peleg (2006), per risolvere i conflitti che si protraggono nel tempo è necessario l'intervento di una terza parte che svolga il ruolo di mediatore. Il peace journalism, secondo Peleg, è in grado di svolgere questo compito permettendo alle parti di conoscersi, rinforzando comprensione ed empatia, orientandosi alle persone e alla ricerca di soluzioni per affrettare la risoluzione di contesti conflittuali.

\section{Giornalismo e migrazioni}


Lo studio dei modi in cui i mezzi di informazione presentano notizie relative ai movimenti migratori è un filone ben radicato nelle scienze sociali (Fotopoulos e Kaimaklioti, 2016). In queste analisi si possono identificare almeno tre punti di osservazione differenti, variamente sovrapposti: la distinzione tra tabloid e quality press, il tipo di fonte utilizzato e l'individuazione delle cornici impresse a queste informazioni. Tutti e tre questi punti di osservazione attraverso cui si analizza la copertura informativa sui movimenti migratori e i migranti si intrecciano alla proposta di Kalfeli $(2018,2019)$ rispetto alle caratteristiche del peace journalism.

Analizzare le informazioni sulle migrazioni attraverso la distinzione tra stampa di qualità e stampa popolare rinforza la nostra decisione di fare una ricerca solo sulla quality press italiana; infatti, considerato che è proprio questo tipo di stampa a offrire un'informazione più accurata rispetto al tema qui in oggetto, rappresenta quella che più può avvicinarsi (e adattarsi) alla proposta presentata dal peace journalism. Su questa distinzione tra la copertura della quality press e dei tabloid, Gabrielatos e Baker (2008) affermano che l'insieme delle informazioni rispetto ai RASIM sia decisamente cresciuto nel decennio dal 1996 al 2005, ma rilevano che la quality press, nel descriverlo, utilizzi una maggiore varietà di termini e una loro più frequente connotazione positiva. Questi risultati si ritrovano nell'analisi di Scott (2009) che si concentra sulle notizie, non solo attinenti al fenomeno migratorio, pubblicate dalla stampa britannica a proposito dei paesi africani. Anche qui si evidenzia la differenza tra giornali di qualità e stampa popolare. Queste differenze si manifestano nella quantità delle notizie, nel numero dei paesi considerati e nei temi trattati (nel primo caso si trovano più hard news, notizie che riguardano politica ed economia). Inoltre, conclude lo studioso, la rappresentazione dell'Africa non è negativa e banalizzata. A risultati pressoché analoghi arriva Gorp (2005), il quale, in una ricerca rispetto al sistema dei media belga, evidenzia come i tabloid si distinguano per una più frequente stigmatizzazione dei richiedenti asilo, definiti sovente come intrusi. Relativamente a migrazioni che avvengono all'interno del contesto dell'Unione europea, Hoops, Thomas e Drzewiecka (2016) si sono focalizzati sulla copertura della stampa inglese relativa al flusso di migranti polacchi. I risultati dimostrano che mentre i tabloid insistono sulla rappresentazione dei migranti come 1" "altro" percepito come una minaccia, la quality press attacca esplicitamente gli atteggiamenti xenofobi adottati dai tabloid, rispecchiando così una delle caratteristiche principali del peace journalism, quella finalizzata a un atteggiamento di critica alle rappresentazioni stereotipate.

Dal secondo punto di osservazione, quello relativo alle fonti di informazione utilizzate dai giornalisti, il lavoro più influente è proprio 
Pre-Print version dell'articolo - Garusi, D., Kalfeli, N., \& Splendore, S. (2020). Senza

nome, senza voce. Il peace journalism come prospettiva per valutare la copertura

informativa italiana dei migranti. MONDI MIGRANTI. https://doi.org/10.3280/MM2020$\underline{002005}$

dovuto a Galtung e Ruge (1965). A seguito di un'analisi incentrata sulla copertura delle crisi del Congo, di Cipro e di Cuba da parte di quattro giornali norvegesi, i due autori presentano risultati che evidenziano come la tendenza è quella di riportare solo le azioni compiute dalle élite del paese. L'altra evidenzia che offrono, in quello che poi la sociologia dei media e gli studi sulla comunicazione definiscono come news values, è che un'occorrenza ha più probabilità di essere coperta quanto più è alta la possibilità di essere restituita in chiave domestica e nazionale. Detto in altri termini, si tende a parlare di migranti solo se arrivano nel paese dal quale scrive l'organo di informazione considerato e lo si fa prevalentemente in chiave nazionale (utilizzando perciò fonti locali).

Questo aspetto di valorizzazione delle fonti ufficiali domestiche, o linguisticamente e culturalmente più vicine, è ben identificabile nella stampa statunitense, in particolare quella collocata negli stati più vicini al confine con il Messico, o in zone in cui sono già insediate comunità ispanoamericane. Larson (2006), in un approfondimento sulla presenza della comunità ispanica statunitense nei media USA, segnala che essa non riceve una copertura proporzionata alla sua ampiezza. Inoltre, questa copertura è intrisa di stereotipi: i migranti messicani sono rappresentati come dannosi per l'economia, per la salute e per la sicurezza. Per contro, la stampa in lingua spagnola diffusa negli Stati Uniti d'America prova a risollevare l'immagine della comunità ispanica, restituendo una maggior vivacità e pluralità in termini di fonti. In uno studio più recente Thorbjørnsrud e Figenschou (2016), attraverso un'analisi del contenuto rispetto all'immigrazione irregolare verso gli Stati Uniti, confermano che l'accesso alle notizie è iniquo e dipendente dallo status e dalla posizione coperta. Cionondimeno, Thorbjørnsrud e Figenschou trovano nelle notizie la presenza di un gruppo che definiscono di fonti marginalizzate, che appare però presente nella informazione in maniera crescente (350-351): sempre più frequentemente per parlare di immigrazione vengono interpellati gli stessi migranti, abbracciando in questo modo il principio del peace journalism che ritiene necessario riportare anche i loro punti di vista quando vengono trattati argomenti che li interessano direttamente.

In un'ampia analisi comparata che ha considerato sia la stampa proveniente da paesi africani, sia quella da paesi europei, Susanne Fengler e colleghi (2018) evidenziano che la stampa africana presta poca attenzione ai singoli cittadini e ai migranti, mentre maggiore enfasi è posta sulle istituzioni e le autorità; parimenti, utilizzando principalmente fonti ufficiali, la stampa europea si focalizza su questioni legate alla sicurezza, trascurando il contesto africano e i suoi attori. 
Pre-Print version dell'articolo - Garusi, D., Kalfeli, N., \& Splendore, S. (2020). Senza

nome, senza voce. Il peace journalism come prospettiva per valutare la copertura

informativa italiana dei migranti. MONDI MIGRANTI. https://doi.org/10.3280/MM2020-

$\underline{002005}$

Il terzo punto di osservazione individuato, già evocato anche nella trattazione compiuta finora, è quello che distingue il frame attribuito alla copertura rispetto ai RASIM. In questa prospettiva si collocano la maggior parte delle ricerche compiute sulla copertura dell'immigrazione. Quello che qui si evidenzia è una radicata propensione della stampa a incorniciare negativamente i migranti e il movimento migratorio. Come dimostra lo stesso studio di Fengler e colleghi (2018), la varietà delle ricerche che appurano questa evidenzia è trasversale rispetto alla nazionalità dei sistemi dei media considerati.

Lo studio di Danso e McDonald (2001) evidenzia una rappresentazione molto negativa del fenomeno migratorio data dai media sudafricani. Gli studiosi si sono concentrati sui giornali del Sudafrica al fine di studiare come venisse rappresentato il flusso di migranti provenienti dai paesi limitrofi nel periodo post-apartheid. Essi hanno osservato che gli articoli, oltre ad essere caratterizzati da una costante ricerca del sensazionalismo, non restituiscono al lettore dettagli sulla complessità del fenomeno migratorio. McDonald e Jacobs (2005) hanno riproposto lo stesso studio per individuare se si fossero verificati dei cambiamenti nella stampa sudafricana rispetto ai temi della xenofobia e delle migrazioni. In questo studio estendono l'analisi anche a giornali di diversi paesi della regione meridionale dell'Africa. Dai dati emerge che la cornice stigmatizzante - contraddistinta da una forte vena xenofoba - è comune agli organi di stampa di Botswana, Zambia e Namibia. Parziale eccezione è rappresentata dallo Zimbabwe e dal Sudafrica dove, a differenza degli altri paesi considerati, si restituisce un quadro almeno polarizzato.

La situazione non cambia neppure in paesi espressione di un sistema dei media considerato generalmente molto professionalizzato (ciò significa con codici deontologici identificabili e generalmente seguiti dai giornalisti). Adeyanju e Neverson (2007) esaminano la copertura dei quattro maggiori giornali canadesi rispetto al caso di una donna congolese sospettata di essere affetta da ebola. Lo studio illustra come la stampa canadese abbia sfruttato il caso per problematizzare l'immigrazione attraverso il richiamo a potenziali rischi per la salute tramite una sistematica associazione tra differenze razziali e rischio. Secondo gli studiosi, i media hanno attivato meccanismi di panico morale attraverso l'uso fallace delle parole nei titoli e nel corpo degli articoli (alla paziente, infatti, non è mai stata diagnosticata ufficialmente l'ebola, eppure il termine è stato sovente usato nella copertura dell'evento).

Anche dal lavoro di Ratajczak e Jędrzejczyk-Kuliniak (2016) sulla rappresentazione dei migranti nella stampa polacca si evidenzia il ricorso a immagini stereotipate. Nel loro lavoro indicano la sovrapposizione tra l'uso della parola rifugiati e quella di immigrati; inoltre mettono in luce 
l'approccio conflittuale ("noi contro loro") attraverso cui sono raccontate le questioni legate all'immigrazione.

In alcuni di questi studi si nota poi che questo tipo di copertura riesce a influenzare l'agenda delle politiche pubbliche: una determinata cornice imposta all'informazione sull'immigrazione spinge i politici a prendere determinate scelte. Questo è un fenomeno noto in comunicazione politica come agenda setting (cfr. Vliegenthart et al., 2016), che si replica anche nel contesto dei temi legati all'immigrazione (Ibrahim, 2005).

Questa rassegna della letteratura suggerisce che, con la parziale eccezione di alcuni organi di stampa rientranti nella categoria della quality press, molta stampa - indipendentemente dalla nazionalità e dall'oggetto della copertura informativa - tende a raccontare il fenomeno migratorio con quello che Kalfeli $(2018,2019)$ definisce la cornice del conflitto: assenza della voce dei migranti, discorso costruito su due parti ("noi e loro"), immigrati sovente rappresentati come un problema, uso di stereotipi e attenzione agli eventi negativi.

La ricerca che di seguito presentiamo ha come obiettivo innanzitutto valutare che tipo di copertura è riservata dalla stampa di qualità italiana ai temi legati all'immigrazione. Successivamente valuteremo questa copertura rispetto alla cornice qui discussa (quella del peace journalism e il suo opposto, quella del conflitto): la copertura informativa italiana che qui rileviamo a quale dei due ideal-tipi si avvicina?

\section{Metodologia della ricerca}

La ricerca di cui qui si dà conto è inclusa in un progetto comparativo più ampio, coordinato da Susanne Fengler, che comprende gli organi di stampa di 17 paesi europei oltre alla Tunisia e agli Stati Uniti d'America. Questo articolo presenta l'analisi solo sulla parte italiana. I due giornali scelti per l'Italia sono il Corriere della Sera e La Stampa, selezionati come due dei quality paper più venduti in Italia ${ }^{6}$. La scelta de La Stampa rispetto a quella di La Repubblica è stata compiuta perché l'archivio utilizzato nella selezione degli articoli (LexiNexis) non includeva quest'ultima.

Sono stati selezionati tutti gli articoli che nel titolo o nell'attacco ${ }^{7}$ contenevano le seguenti parole chiave (con i loro derivati):

\footnotetext{
${ }^{6}$ Si vedano i dati Audipress del primo ciclo 2018, disponibili online attraverso il sito www.primaonline.it, sezione dati.

${ }^{7}$ Si definisce attacco la parte iniziale di un articolo di giornale, quella che nelle pratiche più consolidate di produzione giornalistica dovrebbe aiutare alla comprensione del contenuto e invitare alla lettura.
} 
Pre-Print version dell'articolo - Garusi, D., Kalfeli, N., \& Splendore, S. (2020). Senza nome, senza voce. Il peace journalism come prospettiva per valutare la copertura informativa italiana dei migranti. MONDI MIGRANTI. https://doi.org/10.3280/MM2020$\underline{002005}$

- rifugiat*;

- *migra* (emigrazione, immigrati, migrante, migranti ....);

- $\quad$ asil*.

Gli articoli sono stati individuati all'interno di sei settimane scelte nel periodo tra il 9 agosto 2015 e il 25 marzo 2018. Sono state selezionate le seguenti settimane:

- $\quad 31$ agosto - 6 settembre 2015 ;

- 9 novembre - 15 novembre 2015;

- 4 gennaio - 10 gennaio 2016;

- 9 ottobre - 15 ottobre 2017 ;

- 11 dicembre -17 dicembre 2017;

- 19 febbraio - 25 febbraio 2018 .

La scelta è caduta sulle settimane in cui, nel complesso della ricerca comparata, si attestava un scarto maggior nel numero di articoli rispetto alla settimana precedente. A seguito della scrematura eseguita con i criteri sopraindicati sono stati selezionati 95 articoli: 56 del Corriere della Sera e 39 de La Stampa.

Su questo corpus è stata condotta un'analisi del contenuto. Come unità di analisi è stata considerata l'interezza dell'articolo per i quali sono stati rilevati elementi oggettivi come la presenza di commenti, il riferimento a questioni domestiche, la destinazione e la provenienza dei movimenti migratori di cui si dà conto, i motivi per cui avvengono. In seguito quegli stessi articoli sono stati letti per individuare la prevalenza di una prospettiva conflittuale o di quella del peace journalism, facendo riferimento agli indicatori già discussi e proposti da Kalfeli $(2018,2019)$ (vedi tabella 1 sopra).

Gli articoli sono stati analizzati da due coder che hanno lavorato in stretto contatto per l'interpretazione del codebook, il quale stabiliva l'insieme delle linee guida per eseguire l'analisi. Il $20 \%$ degli articoli è stato poi analizzato da un terzo coder per decretare il coefficiente di affidabilità dell'analisi che è risultato oltre il .90 , valore che possiamo considerare più che soddisfacente (Neuendorf, 2002: 145).

Senza voce, senza nome, senza motivi, senza provenienza: i RASIM nella stampa italiana 
Rispetto agli articoli selezionati i due giornali offrono una copertura simile. Tuttavia si può individuare una differenza: La Stampa dedica un più alto numero di articoli al contesto estero o internazionale, mentre nella quasi totalità degli articoli del Corriere della Sera gli eventi riportati hanno un legame esclusivo con l'Italia. Il quotidiano torinese pone maggiore attenzione ai movimenti migratori verso altri paesi dell'Unione europea, mentre il Corriere si concentra principalmente su quelli diretti in Italia. La spiegazione rispetto a questa differenza si può ricondurre alla direzione del giornale torinese durante il periodo in cui è stata svolta la ricerca, quella di Maurizio Molinari, ex corrispondente da New York, Bruxelles e Gerusalemme, il quale ha impresso al giornale una più spiccata propensione alle questioni internazionali.

La maggior parte degli articoli analizzati sono focalizzati sul racconto degli avvenimenti, solo 4 di essi - tutti pubblicati dal Corriere della Sera restituiscono opinioni e valutazioni dei giornalisti. In entrambe le testate è prestata scarsa attenzione alle specifiche motivazioni che hanno spinto $i$ migranti a spostarsi (77 degli articoli analizzati non ne fa menzione). L'unico riferimento presente è quello alla generica richiesta di asilo politico. Insomma, non sono discusse le motivazioni che spingono i RASIM a partire, né sono presenti chiarimenti riguardo alla natura delle diverse richieste di asilo. In merito al paese di origine dei migranti, nella maggior parte degli articoli (72) non è specificata la loro provenienza. In linea con lo scarso livello di dettaglio con cui sono state riportate le motivazioni e le origini dei migranti, solo in una manciata di articoli l'attore principale è un migrante individuabile singolarmente (4) o un ristretto e identificabile gruppo di questi (3), come ad esempio una famiglia. Invece, la maggior parte delle volte in cui la notizia ruota attorno ai RASIM essi sono rappresentati come un gruppo anonimo e indefinito, migrazioni e migranti sono trattati come categoria generica. Nella quasi totalità degli articoli analizzati (circa 80) non è mai citato il nome e la provenienza dei migranti di cui si parla.

La prospettiva più utilizzata per raccontare l'immigrazione è quella legata al dibattito politico domestico o europeo ( 37 articoli), prospettiva che esclude quasi sistematicamente il punto di vista dei migranti (nella quasi totalità dei casi nessun migrante viene rappresentato individualmente per nome o per paese di provenienza).

Tra tutti gli articoli presi in considerazione in questo lavoro, poco meno della metà va oltre la logica "noi e loro". Il "noi" è generalmente richiamato da forze istituzionali, o più precisamente politico partitiche, che focalizzano la loro attenzione sull'immigrazione come problema. Due esempi non italiani sono esemplificativi di ciò. Il primo è relativo alle parole di un rappresentante dell'Unione Cristiano-Sociale in Baviera (CSU) che 
Pre-Print version dell'articolo - Garusi, D., Kalfeli, N., \& Splendore, S. (2020). Senza

nome, senza voce. Il peace journalism come prospettiva per valutare la copertura

informativa italiana dei migranti. MONDI MIGRANTI. https://doi.org/10.3280/MM2020$\underline{002005}$

proponeva di: "introdurre un limite massimo annuale al numero degli ingressi di rifugiati nel Paese" a causa della minaccia perpetrata ai "vecchi valori di «patria», «famiglia»e «tradizione» messi in pericolo dall'ondata migratoria e dall'ideologia sessantottina della società multiculturale" ( $L a$ Stampa, 11 ottobre 2017, p. 3). Il secondo riguarda i leader dei quattro paesi di Visegrad che: "intendono contribuire [...] ad allontanare i migranti alle frontiere e fuori dai Paesi Ue. Il premier ungherese Viktor Orbán ha parlato di «combattimento» per «respingere con successo gli attacchi»" (Corriere della Sera, 16 dicembre 2017). Questi non sono esempi sparuti, un buon numero di articoli contiene questo tipo di posizioni tenute da vari esponenti politici. Nella radicata pratica del giornalismo di dare voce alle fonti istituzionali, il risultato è quello della restituzione del discorso politico anche nelle sue forme più estremiste e conflittuali che rafforzano il frame del "noi contro loro".

In questo tipo di articoli, anche quando il conflitto è attenuato, solo raramente la loro voce è presa in considerazione (detto diversamente, raramente i migranti sono citati direttamente oppure sono usate anche fonti istituzionali che hanno a che fare con i loro paesi di origine). Questo accade sia quando si parla di politiche a livello europeo, sia quando si raccontano episodi a livello iper-locale. Ad esempio, in un articolo del Corriere della Sera (15 dicembre 2107) intitolato "Migranti, dialogo tra sordi in Europa; Merkel con l'Italia, ma è stop alle quote", riguardante la ripartizione dei rifugiati e la riforma del Trattato di Dublino, sono utilizzati come fonte Macron, Merkel, Gentiloni, Tajani e la Mogherini, ma nessuna altra voce extra europea viene chiamata in causa. Come esempio a livello iper-locale si può menzionare un articolo pubblicato il 21 febbraio 2018, relativo alle proteste dei cittadini di un comune in provincia di Lecco per l'apertura di un nuovo centro di accoglienza profughi.

Ma i cittadini non sembrano fidarsi: «Chiediamo all'amministrazione di fare tutte le verifiche del caso per sapere come stanno realmente le cose. Abbiamo informazioni fidate in merito al fatto che ci sarebbe una trattativa in corso. Non è una questione di razzismo, ma l'area della stazione presenta già troppi problemi dal punto di vista della sicurezza, questo sarebbe il colpo di grazia», rincara la dose Cristina Valsecchi, titolare di una gelateria. E le parole della proprietà, che non smentisce e non conferma, non servono certo a rasserenare gli animi: «A casa mia faccio quello che voglio», risponde piccato Carlo Colombo, della Colombo manutenzione stabili, rimarcando che «se il piano di lottizzazione per riqualificare l'intera area presentato a suo tempo al Comune non fosse irrimediabilmente fermo al palo, la zona a quest'ora avrebbe già un altro volto». (Corriere della Sera, 21 Febbraio 2018, p. 14).

La voce dei migranti emerge quando si dà conto di atti di violenza subita dagli stessi. Accade in un articolo pubblicato da La Stampa 1'11 ottobre 2017 
Pre-Print version dell'articolo - Garusi, D., Kalfeli, N., \& Splendore, S. (2020). Senza

nome, senza voce. Il peace journalism come prospettiva per valutare la copertura

informativa italiana dei migranti. MONDI MIGRANTI. https://doi.org/10.3280/MM2020$\underline{002005}$

che riporta le testimonianze in tribunale di alcuni migranti torturati in un campo di prigionia in Libia. Questa situazione si ripete in un articolo pubblicato sempre da La Stampa (6 settembre 2015) in cui vengono riportate le parole di una migrante che, appena scesa dal treno in una stazione della periferia di Vienna, sollecitata dai giornalisti racconta la sua esperienza:

Non saprei dire il momento peggiore. Scappando dalla Siria, ci hanno sparato. Abbiamo tentato tre volte l'attraversata per l'isola di Kos, prima di farcela. In Macedonia abbiamo camminato 50 chilometri a piedi. Ma l'Ungheria non potrò mai dimenticarla: i poliziotti hanno picchiato mio marito per obbligarlo a lasciare le impronte digitali. Ci urlavano continuamente: "State zitti, state zitti!". Non avevano pietà nemmeno per mio figlio ( $\mathrm{La}$ Stampa, 15 settembre 2015, p. 1).

Questo articolo coglie molte delle caratteristiche del peace journalism: non solo dà voce ai migranti, ma lo fa per raccontare e incorniciare il contesto di provenienza. Secondo i principi del peace journalism, quando i media riportano atti di violenza il focus dovrebbe essere posto sulle vittime e sulla sofferenza da loro patita (Galtung, 2006). Nel contesto della nostra ricerca, gli articoli che più si sono avvicinati a queste caratteristiche, e quelle proposte da Kalfeli $(2018$, 2019), sono proprio quelli che passano dalle testimonianze dei migranti, capaci di innescare un meccanismo che porta ad avere più attenzione nei confronti delle storie degli esseri umani, a focalizzarsi su qualsivoglia tipo di violenza come problema reale e a sforzarsi nel tentativo di decostruire gli stereotipi.

\footnotetext{
«Sapevo che faceva freddo, ma non così», ha ansimato l'unico che parlottava italiano. «Je ne savais pas la neige», non sapevo la neve, ha espirato il capogruppo, in un francese arrangiato come la sua vita. Il sapere sposta le montagne, ma della montagna questi cinque non sapevano niente. Dalla Costa d'Avorio ai costoni della Scala, i barconi mediterranei e i sotterranei torinesi dove dormire, vent'anni d'età e nessuna idea del mare bianco che li aspettava quassù. Cuori nella tormenta. (La Stampa, 15 settembre 2015, p. 1).
}

Come sostiene Peleg (2006), nei contesti di potenziale conflitto, un approccio empatico verso tutte le parti e una maggiore attenzione al contesto riducono la propensione a prendere una posizione netta.

Se in qualche articolo tra quelli analizzati si intercettano notizie restituite seguendo le caratteristiche dell'ideologia giornalistica del peace journalism, la caratteristica che invece manca totalmente è legata alla decostruzione di stereotipi e in senso più lato al media criticism. Non si trovano articoli in cui i due giornali si interrogano sul modo scorretto di coprire l'immigrazione.

\section{Conclusione}


Questo articolo rappresenta una proposta normativa per introdurre sia nel dibattito scientifico italiano, sia - ed eventualmente - nelle pratiche di produzione giornalistica rispetto ai temi dell'immigrazione, un approccio da peace journalism. Questa proposta è stata qui valutata rispetto a un'analisi del contenuto compiuta su una selezione di articoli di informazione sul tema delle migrazioni pubblicati dal 2015 al 2018; gli articoli sono stati selezionati da due giornali italiani che possiamo annoverare nella categoria della quality press: il Corriere della Sera e La Stampa. La scelta è motivata dal fatto che la rassegna della letteratura indica proprio in questo tipo di giornali una maggiore presenza del tema e una rappresentazione più varia in termini della qualità degli articoli offerti (Scott, 2009; Gabrielatos e Baker, 2008). In sostanza, considerando il peace journalism come una buona proposta per trattare questioni legate all'immigrazione, abbiamo indagato la sua presenza proprio nei giornali considerati più pronti ad accoglierlo.

Due le conclusioni principali emerse dalla ricerca. Rispondendo alla domanda di ricerca per come l'abbiamo formulata alla fine della rassegna della letteratura, la prima conclusione rileva che la copertura informativa italiana si avvicina di più al frame del conflitto. Emerge un ricorso a fonti istituzionali che spesso restituisce una retorica conflittuale ("noi contro loro"), soprattutto quando la fonte sono determinati partiti, o che affronta l'immigrazione come un problema (ad esempio le istituzioni europee quando discutono rispetto alla ripartizione dei rifugiati). Inoltre, per radicate routine produttive, ma probabilmente anche per la sensibilità del fruitore dell'informazione, i giornali non raccontano il fenomeno migratorio nella sua globalità, ma utilizzano cornici locali. In questi articoli i giornalisti riportano le informazioni non mostrando né sostegno né disapprovazione a quanto riportato, per dirla con David Ryfe (2012: 11), lo fanno seguendo uno dei principi del giornalismo: coprono la storia, senza diventare la storia.

La seconda conclusione è che gli articoli che contengono le voci dei migranti, che contestualizzano il percorso di emigrazione e immigrazione, $o$ che danno conto delle motivazioni per cui hanno deciso di emigrare, non includono l'accezione conflittuale "noi contro loro".

Se molti dei tratti distintivi dell'informazione qui analizzata non possono essere agevolmente superati (per esempio l'esigenza di valorizzare informazioni che sono più vicine al contesto nazionale), nelle pratiche giornalistiche si dovrebbe invece insistere rispetto all'esigenza di inserire una pluralità di fonti, specie quelle che Thorbjørnsrud e Figenschou (2016) definiscono "emarginate", riferendosi in particolare alla voce degli stessi migranti. 
Pre-Print version dell'articolo - Garusi, D., Kalfeli, N., \& Splendore, S. (2020). Senza

nome, senza voce. Il peace journalism come prospettiva per valutare la copertura

informativa italiana dei migranti. MONDI MIGRANTI. https://doi.org/10.3280/MM2020-

$\underline{002005}$

Applicando una lettura più interpretativa e meno rigorosa rispetto ai risultati di ricerca, il secondo - sparuto - gruppo di articoli che motiva la seconda conclusione, spiega perché Galtung (1969) sostenga che il modo in cui i media presentano il conflitto può essere il principale fattore nel determinare come questo proseguirà, se si perpetuerà o meno. Anche negli stralci sopra riportati traspare quanto alcuni tipi di articoli rafforzino e alimentino il conflitto e quanto altri tipi sprigionino invece un potenziale effetto calmierante. Come sostiene Peleg (2006), i conflitti che si protraggono nel tempo necessitano una terza parte che svolga il ruolo di mediatore, che faciliti l'interazione tra le parti in causa. Con questo tipo di articoli, come Peleg suggerisce, il peace journalism ha buone possibilità di svolgere questo ruolo, perché permette alle parti di conoscersi agevolando comprensione ed empatia e riducendo la stigmatizzazione dei diversi soggetti coinvolti.

L'introduzione o il rafforzamento dei dispositivi, delle pratiche e dei meccanismi che mettono in opera il peace journalism, è però innanzitutto una svolta culturale. Qui si intende questa svolta culturale proprio nei termini discussi nei paragrafi precedenti (Hanitzsch, 2007a; ma si veda anche l'accezione di Ryfe, 2012), come tentativo preciso di inserire il peace journalism all'interno della cultura giornalistica italiana, farlo diventare una ideologia giornalistica ben riconoscibile e posizionata all'interno dell'arena giornalistica nazionale. Certo, non è uno sforzo immediato, né tanto meno semplice. Lo stesso Hanitzsch (2007b) sostiene che l'introduzione pratica del peace journalism è generalmente fondata su una prospettiva volontaristica $\mathrm{e}$ individuale; una sua introduzione strutturale comprende la possibilità che $\mathrm{i}$ giornalisti abbiano il tempo e le risorse per creare l'accesso al numero e alla qualità di fonti adeguata. Nella contemporanea ecologia dei media, le logiche con cui sono confezionate le notizie rappresentano una limitazione ulteriore alla diffusione del peace journalism: velocità, necessità di attrarre larghe fette di pubblico, esigenze commerciali sono un detrimento al suo inserimento e sviluppo.

Questa svolta qui evocata passa anche dallo sforzo di sostenere il peace journalism da parte dei diversi stakeholder che sono interessati a far sì che circoli una più accurata informazione sui migranti: le associazioni, le organizzazioni no profit, le università, i cittadini. Il peace journalism dovrebbe diventare il termine di paragone, la cartina di tornasole, l'oggetto di dibattito sui modi in cui il giornalismo racconta i temi dell'immigrazione. Il peace journalism dovrebbe diventare il dispositivo per decostruire e contestare le rappresentazioni stereotipate. La mancanza di questo approccio, almeno stando ai risultati della nostra ricerca, stupisce se considerato il ruolo della Carta di Roma. Esistono infatti molte analogie tra diversi passaggi della 
Pre-Print version dell'articolo - Garusi, D., Kalfeli, N., \& Splendore, S. (2020). Senza nome, senza voce. Il peace journalism come prospettiva per valutare la copertura informativa italiana dei migranti. MONDI MIGRANTI. https://doi.org/10.3280/MM2020$\underline{002005}$

Carta e i principi del peace journalism: l'esortazione a non diffondere "informazioni imprecise, sommarie o distorte", a "interpellare [...] esperti ed organizzazioni specializzate in materia" e i richiami non solo a restituire i fatti, ma anche ad analizzare in modo critico il contesto; principio cardine del peace journalism (Galtung 1998; Lynch 2017; Kalfeli e Frangonikolopoulos 2019). Questa ricerca suggerisce che esiste una fessura, un passaggio obbligato, per restituire un'informazione diversa rispetto ai fenomeni migratori, ed è dare la voce ai migranti, ascoltare e raccontare le loro storie. Anche da un punto di vista strettamente giornalistico, molti degli articoli analizzati in questa ricerca rivelano quali ipnotici dispostivi drammaturgici siano il racconto delle storie di molti migranti. Il peace journalism rivendica sicuramente la necessità normativa di mettere in agenda le storie dei migranti attraverso le loro fonti, ma rivela anche che queste storie ben si adattano a diffuse cifre stilistiche del giornalismo contemporaneo come ai suoi più consolidati valori notizia.

\section{Bibliografia}

Adeyanju, C. T., \& Neverson, N. (2007). «There Will Be a Next Time»: Media Discourse about an «Apocalyptic» Vision of Immigration, Racial Diversity, and Health Risks. Canadian Ethnic Studies, 39(1-2): 79-105. https://doi.org/10.1353/ces.0.0003

Bentivegna, S., Natale, P. (2019). La forza di una campagna debole. Il caso delle elezioni politiche 2018. Comunicazione Politica, 1: 5-14. DOI: 10.3270/93026

Binotto, M., Bruno, M., Lai V. (2016). Tracciare confini. L'immigrazione nei media italiani. Milano: Franco Angeli.

Bratic, V., Ross, S. D., Kang-Graham, H. (2008). Bosnia's Open Broadcast Network: A Brief but Illustrative Foray into Peace Journalism Practice. Global Media Journal, 7(13). Testo disponibile al sito: http://www.globalmediajournal.com/openaccess/bosnias-open-broadcast-network-a-brief-but-illustrative-foray-into-peacejournalism-practice.php?aid=35218, 25/09/2019.

Bruno, M. (2014). Cornici di realtà. Il frame e l'analisi dell'informazione. Roma: Guerini.

Chadwick, A. (2013). The Hybrid Media System: Politics and Power. Seconda Edizione. Oxford Studies in Digital Politics. New York, NY: Oxford University Press.

Danso, R., McDonald, D. A. (2001). Writing Xenophobia: Immigration and the Print Media in Post-Apartheid South Africa. Africa Today, 48(3): 115-137. DOI: $\underline{10.1353 / a t .2001 .0050}$

Deuze, M. (2005). What Is Journalism? Professional Identity and Ideology of Journalists Reconsidered. Journalism, 6(4): 442-464. https://doi.org/10.1177\%2F1464884905056815

Diamanti, I. (2016). Invasione mediale degli immigrati senza volto. In: Carta di Roma, a cura di, Notizie oltre i muri, IV Rapporto Carta di Roma, pp. 9-13.

Fengler, S., Bastian, M., Brinkmann, J., Zappe, AC (2017). The Politicization of the Migrant Crisis: A Comparative Analysis in six European and five African Countries of how the Press covered Migration from Africa to Europe. November 22 - 232017 ECREA PolComm Conference in Zurich, Switzerland. 
Pre-Print version dell'articolo - Garusi, D., Kalfeli, N., \& Splendore, S. (2020). Senza nome, senza voce. Il peace journalism come prospettiva per valutare la copertura informativa italiana dei migranti. MONDI MIGRANTI. https://doi.org/10.3280/MM2020$\underline{002005}$

Fotopoulos, S., Kaimaklioti, M. (2016). Media Discourse on the Refugee Crisis: On what have the Greek, German and British Press Focused? European View, 15(2): 265-279. https://doi.org/10.1007/s12290-016-0407-5

Gabrielatos, C., Baker, P. (2008). Fleeing, Sneaking, Flooding: A Corpus Analysis of Discursive Constructions of Refugees and Asylum Seekers in the UK Press, 1996-2005. Journal of English Linguistics, 36(1): 5-38. https://doi.org/10.1177/0075424207311247

Galtung, J. (2006). Peace journalism as an ethical challenge. Global Media Journal: Mediterranean Edition, 1(2): 1-5. Testo disponibile al sito: https://eirineftikidimosiografia.files.wordpress.com/2013/11/galtung-j-peace-journalismas-an-ethical-challenge.pdf, 25/09/2019.

Galtung, J. (1998). High road, low road: charting the course for peace journalism. Track Two: Constructive Approaches to Community and Political Conflict, 7(4): 95-102. https://doi.org/10.1007/978-3-642-32481-9_8

Galtung, J. (1969). Violence, Peace, and Peace Research. Journal of Peace Research, 6(3), 167-191. https://doi.org/10.1177/002234336900600301

Galtung, J., Ruge, M. H. (1965). The Structure of Foreign News. The presentation of the Congo, Cuba and Cyprus crises in four Norwegian Newspapers. Journal of International Peace Research, 2(1): 64-91. https://doi.org/10.1177/002234336500200104

Godler, Y., Reich, Z. (2017). Journalistic Evidence: Cross-Verification as a Constituent of Mediated Knowledge. Journalism, 18(5): 558-574. DOI: 10.1177/1464884915620268

Gorp, B. (2005). Where is the Frame? European Journal of Communication, 20(4): 484507. https://doi.org/10.1177/0267323105058253

Hanitzsch, T. (2007a). Deconstructing Journalism Culture: Toward a Universal Theory. Communication Theory, 17(4): 367-85. DOI: 10.1111/j.1468-2885.2007.00303.x

Hanitzsch, T. (2007b). Situating peace journalism in journalism studies: A critical appraisal Conflict \& Communication Online, 6(2): 9. Testo disponibile al sito: http://cco.regeneronline.de/2007_2/pdf/hanitzsch.pdf, 25/09/2019.

Hanitzsch, T. (2004). Journalists as peacekeeping force? Peace journalism and mass communication theory. Journalism Studies, 5(4): 483-495. https://doi.org/10.1080/14616700412331296419

Hoops, J. F., Thomas, R. J., Drzewiecka, J. A. (2016). Polish 'Pawns' between nationalism and neoliberalism in British newspaper coverage of post-European Union enlargement polish immigration. Journalism, 17(6): 727-743. https://doi.org/10.1177/1464884915585960

Ibrahim, M. (2005). The Securitization of Migration: A Racial Discourse1. International Migration, 43(5): 163-187. https://doi.org/10.1111/j.1468-2435.2005.00345.x

Kalfeli, N. (2019). Beyond the Us/Them Binary: An Analysis of Greek Media's Framing of Immigration through a Peace Journalism Lens. ECREA Journalism Studies Section Conference, Vienna, 14-15 febbraio.

Kalfeli, N. (2018). Diversity and peace journalism: representations of immigration in the Greek press during the Greek financial crisis (Unpublished doctoral dissertation). Aristotle University of Thessaloniki, Thessaloniki, Greece.

Kalfeli, N., Frangonikolopoulos, C. A. (2019). Peace Journalism. The International Encyclopedia of Journalism Studies, pp. 1-5. https://doi.org/10.1002/9781118841570.iejs0139

Larson, S. G. (2006). Media \& Minorities: The Politics of Race in News and Entertainment Rowman \& Littlefield.

Loyn, D. (2007). Good journalism or peace journalism? Conflict \& Communication Online, 6(2): 1-10. Testo disponibile al sito: http://www.cco.regeneronline.de/2007 2/pdf/loyn.pdf, 25/09/2019. 
Pre-Print version dell'articolo - Garusi, D., Kalfeli, N., \& Splendore, S. (2020). Senza nome, senza voce. Il peace journalism come prospettiva per valutare la copertura informativa italiana dei migranti. MONDI MIGRANTI. https://doi.org/10.3280/MM2020$\underline{002005}$

Lynch, J. (2017). What Is Peace Journalism? Transcend Media Service, August 2017. Disponibile online https://www.transcend.org/tms/2017/08/what-is-peace-journalism-3/, 30/10/2019.

McDonald, D. A., Jacobs, S. (2005). (Re)writing xenophobia: Understanding press coverage of cross-border migration in Southern Africa. Journal of Contemporary African Studies, 23(3): 295-325. https://doi.org/10.1080/02589000500274050

McGoldrick, A. (2006). War Journalism and «Objectivity». Conflict \& Communication Online, 5(2): 1-7. Testo disponibile al sito: http://www.cco.regeneronline.de/2006_2/pdf/mcgoldrick.pdf, 25/09/2019.

Neuendorf, K.A. (2002). The content analysis guidebook. Thousand Oaks, CA: Sage.

Peleg, S. (2006). Peace Journalism through the Lense of Conflict Theory: Analysis and Practice. Conflict \& Communication Online, 5(2): 1-17. Testo disponibile al sito: http://www.cco.regener-online.de/2006_2/pdf/peleg.pdf, 25/09/2019.

Pogliano, A. (2011). Nominare mostrando. L'influenza della visibilità sul racconto giornalistico dell'immigrazione. Mondi Migranti, 1: 231-251. https://doi.org/10.3280/MM2011-001011

Pogliano A., Ponzo I. (2019). Asilo e crisi urbane: dinamiche di narrative-making tra media e policy network locali. Mondi Migranti, 1: 119-141. DOI: 10.3280/MM2019-001008

Ratajczak, M., \& Jędrzejczyk-Kuliniak, K. (2016). Muslims and Refugees in the Media in Poland. Global Media Journal, 6(1):1-17. Disponibile online: https://www.dbthueringen.de/servlets/MCRFileNodeServlet/dbt_derivate_00035508/GMJ11_Ratajacza k_Jedrzejczyk-Kuliniak.pdf, 25/09/2019.

Ryfe, D. (2012). Can Journalism Survive? An Inside Look at American Newsrooms. Cambridge: Polity.

Scott, M. (2009). Marginalized, negative or trivial? Coverage of Africa in the UK press. Media, Culture \& Society, 31(4): 533-557. https://doi.org/10.1177/0163443709335179

Splendore, S. (2017). Giornalismo ibrido: come cambia la cultura giornalistica italiana. Roma: Carocci.

Thorbjørnsrud K, Figenschou T.U. (2016). Do marginalized sources matter? Journalism Studies, 17(3): 337-355. DOI: 10.1080/1461670X.2014.987549

Van Aelst, P., Strömbäck J., Aalberg T., Esser F., de Vreese C., Matthes J., Hopmann D., et al. (2017). Political Communication in a High-Choice Media Environment: A Challenge for Democracy? Annals of the International Communication Association, 41(1): 3-27. DOI: 10.1080/23808985.2017.1288551

Vliegenthart, R., Walgrave, S.,. Baumgartner, F.R., Bevan, S., Breunig, C., Brouard, S., Chaqués Bonafont, L. (2016). Do the Media Set the Parliamentary Agenda? A Comparative Study in Seven Countries. European Journal of Political Research, 55(2): 283-301. https://doi.org/10.1111/1475-6765.12134

Waisbord S. (2013). Reinventing Professionalism: Journalism and News in Global Perspectives. Cambridge: Polity. 